\title{
Enterprise Integration using Service Oriented Architecture
}

\author{
Oldooz karimi $^{1}$ and Nasser modiri ${ }^{2}$ \\ ${ }^{1}$ Department of Computer Engineering,MSC,Azad University,Branch Sofiyian \\ sofiyian, Iran, \\ oldooz_karimi@yahoo.com \\ ${ }^{2}$ Department of Computer Engineering,Phd,Azad University,Branch zanjan \\ Tehran, Iran, \\ nassermodirietyahoo.com
}

\begin{abstract}
Today, one of the new solutions to build organizational systems applications, service-based solution is considered. This paper will attempt to identify with the full service approach we offer Integration. In this method, a process model of organization and inventory lists organizations as inputs received during a three-step process for integrating services organizations are recognized organization. Try this method of communication between services is possible and declined to increase communication within the service.
\end{abstract}

\section{Keywords}

Integration, Service Oriented Architecture, Enterprise.

\section{Introduction}

Match the environment and respond quickly to customer needs, the main challenge today as forward is one of the organizations. Today, one of the new solutions to build organizational systems applications, service-based solution is considered. In addition, service-based architecture as one of the architectural style ahead in the organizational architecture solutions are proposed. Concept of service oriented architecture service agreement with the capabilities needed to bring the organization provides. More generally, in this paper a research project to develop and offer a new method for extracting and identifying the steps the service life cycle model of serviceoriented architecture is service. This paper will attempt to identify with the full service approach we offer Integration. In this method, a process model of organization and inventory lists organizations as inputs received during a three-step process for integrating services organizations are recognized organization. Try this method of communication between services is possible and declined to increase communication within the service.

\section{2. later works}

According to some existing methods for extraction and identification of service with them, and identify general weaknesses of this method and classified in a table, my recommendation is that research and studies to make the next fix weaknesses in existing methods and design be defined and implemented new procedures to the future not far from the result of research carried out, comprehensive approach in this area and provided should be presented.

Here is a list of some things in the future if researchers can get by offered:

1.Provide a way to evaluate other factors in service quality and establish the service described phases.

2. Provide a method for making factor in terms grain service, service design process. 
3. Provide a way to define the exact scope and functional service territory. However, challenges in the field of service-oriented architecture in this article that also mentioned some of them were, it seems a good potential for research and research exist. Therefore, dear students and researchers studying various aspects of the challenges can be referred to appropriate areas for research to find.

\section{Integration Application}

One of the main orientations of information technology, integration and connectivity of information systems and technologies to achieve seamless and integrated systems to Means, the environment needed to achieve the information society will also. Due to this need, this paper provides the framework for evaluating new technologies EAI capabilities in order to select based on integration technologies necessary steps are taken. Our extensive literature review of EAI and provide a new framework in the field of technology assessment of EAI, the existing frameworks we have developed.

Development of existing frameworks is significant from several aspects and it is required for the efficient sharing and integrated data, applications and organizational processes in an organizational context.

The relationship between information systems, software and various organizational new kind of virtual environment is associated. Include "context" and "architecture" needed to engage the organizational ability of a system around the year 2004 was considered. A close relationship with the Business Process Management (BPM) is. Because BPM based on the implementation and management of integration processes and formed a work flow process of the various organizational units and related systems and requires them to cross the use of information and organizational components of the system are varied. And proposed approaches to organizations:

- Although service oriented architecture advantages of other approaches, but since most organizations can not step in a complex infrastructure systems and change your words and being a service oriented and multi-year evolution is therefore proposed solution to the organization Use service-oriented architecture, integration adapters with non-service oriented systems. Thus, until all the systems to service-oriented organization, connectivity and service oriented systems interact with the new system will also hereditary.

\section{Service oriented architecture and integration challenges associated with:}

Service Oriented Architecture (SOA) as a method for information systems architecture to allow organizations to changes in the conventional business world to increase their flexibility.

For the development of service oriented systems based on ideas presented in different ways in which all that the first step is modeling services. The first step in professional services required to cover the needs identified and then these services are designed as exact. Thus, step service diagnosis as first step in the development of service oriented systems is important because diagnostic services with appropriate quality level in this step led to the development of high quality and flexible system that increases flexibility to changing environment in business organizations and work will help a lot.

Design problem, we do different methods for identifying services offered step. Because the process is usually a large number of organizations, organizational support, diagnostic services organizations, manual work difficult, time consuming and error prone to be.

Overall eight quality characteristics are expected for the service [1] which includes: capability of reusing, having marked the official contract, having high level of abstraction, autonomy, loose connection between the service composition capability, and the situation without being detectable. Should be noted that all these features can not be identified in the service of steps between them and the only loose connections between the features or use of this service, ability to 
combine and limit autonomy in this step are to be measured and evaluated [2]. Another feature that should be considered in services was identified, grain service. This feature on some characteristics of the final system, including the efficiency and flexibility of the system is effective.

Therefore, our service method of identifying common organizational models used as input to the input and we need not be complicated and unconventional. Common models for organizational models that form the common characteristics of different organizations are used and their preparation is a complex process and timing characteristics of the loose connections between service quality, reuse, composition and ability to be taken into account the autonomy and range of services that define the specific requirements in addition to covering all of the organization, the qualitative characteristics of possible increases. And according to service-oriented architecture challenges that we in the following:

1)Organizational challenges of system integration (EAI) and the interaction between organizational ability (B2B)

2) technology challenges in the field of macro-information systems

The most important challenge in the field of information systems architecture is lack of integration and interaction of interoperability information systems (within the organizational / between organizational).

In this regard, information technology strategies typically include:

A) Integration of information systems within the organization, usually under the title (EAI) is known. One of the goals of this architecture is also service oriented. B) Automation processes that the organization is known as BMP and great value for today's organizations to take. C) The interaction ability of systems / systems as among the organizational (B2B) is known. Thus the main question this paper by identifying service characteristics method is above.

\section{Comparison of Service Oriented Architecture and EAI}

Many professionals in the field of integration, service oriented architecture as the next generation of EAI technologies is considered. Traditional EAI technologies for integration applications that have been developed separately, as well as work do. But it should be noted that based methods, specific solutions that EAI vendors to the organization and its platform vendors will depend. Today with Web services become pervasive, as appropriate technology transfer, service oriented architecture Web services as integration solutions based on standards have been discussed. So, you said that being platform-independent Web services, has caused them to use instead of conventional EAI technologies, is extremely attractive. Furthermore, the introduction of bus service organizational products (ESB), integration of web service standards based on the famous and the common super made. Another point is that although the final destination EAI and serviceoriented architecture, implementation of business processes based on cart applications available, but each of them to achieve this goal, the way they used completely different has a root. EAI on putting on display the performance of applications, focuses on the causes, the final model based on cart applications available to take shape. In contrast, SOA applications on the cloak and instead, put on display services set independently of the application is focused. Next, the ESB product and its application in the SOA, with more description are investigated.

ESB in terms of concept, evolved mechanisms to store and send the software products such as message-oriented middleware (MOM) and now EAI technologies with conventional technologies orchestrate construction, Web services, XML and XSLT is combined. ESB can design, develop, deploy and manage SOA provides challenges and making orchestrate services, data synchronization and business activity monitoring (BAM) on the side helps. Physically, ESB backbone needed to implement SOA offers. ESB applications and component integration of discrete, it allows to interact with each other, the total of the services for the formation processes of the business combination has created and thus the processes of business organizations 
automate with the advent of Web services and service oriented architectures, products, EAI, ESB products to place and service-oriented architecture will be less complicated.

\section{6. proposed Service Oriented Architecture for integration of organizational programs}

In this section, the overall process of the proposed method, including phases, activities are presented. Overall process of the proposed method in three-phase service-oriented analysis, evaluation and optimization has been designed and organized.

In the first stage, namely service-oriented analysis phase, the field analysis of business organizations and non-operational and operational performance systems are studied hereditary organization. At the end of this stage of candidate organizations to identify and list services are offered.

Secondly, accuracy is evaluated candidate services. At this stage if necessary and in order to achieve maximum compliance with organizational objectives and achieve good quality, necessary reforms on service and final list of nominations made service organizations is presented. In the third stage, the final service organization based on the nature and logic are defined and the potential, in three layers of processing services, or basic services and application services are classified.

More steps in this section, activities and how to perform administrative activities in each of the proposed method to extract and identify the service is described.

Phase I: Service-Oriented Analysis

Service-Oriented Analysis Phase three is operating. These activities include field analysis of the business organization, systems analysis and presentation of the organization nominated list services organization. Assuming that the first activity of the business organization in the field of analysis and user cases are extracted, activity diagrams related to each case and plotted Sort of processing activities within the charts, candidate services are identified. In the second activity, the operational functions of the existing systems and organizations considered as part of other services are considered candidates. Finally, with the candidate list of services they provide are summarized ID.

Phase II: Evaluation and Optimization

Overall at this stage two major themes will be examined. One of these two candidates of compliance services and other organizational goals and evaluate the quality of services nominated in accordance with the principles of service orientation is. If the service in compliance with organizational goals contradiction exists, or quality of service level is not suitable, necessary reforms at this stage are done on the service logic. At the end of this stage, the final list of service organizations is presented.

Candidate services to control compliance with the goals of business organizations are necessary and the activities carried out. These activities include "tree produced goals," "diagnostic services to the nominated objectives" and "analysis, composition, or creating new services" are.

The main purpose of the allocation of organizational goals nominee services, services to show compliance with organizational goals is identified. If some of the organizational goals by any of the services are not covered by existing candidates, in this case should create new service candidates and are dedicated to the purposes intended. Also, if the service despite the allocation of organizational goals, needs some goals are not fully covered, in this case should also create new service candidates and are dedicated to the purposes intended.

In this section, the principles of service orientation to service quality measures are considered. And also possible to evaluate and assess each of the principles as service quality characteristics have been studied.

Phase III: Organization

During the first phase, a list of candidate services identified through analysis of the business areas of the organization and functions of existing systems were presented. In the second phase, first rate service compliance with the goals of candidates and then evaluated their quality. If there are 
problems to improve service quality and optimizing contract performance logic and services were nominated for acting.

The first phase of final list of organizations provided services are offered. The final services in the three layers of business services, application services and basic services are classified. In this section, the final list of service organizations is presented. Very important point is that the final version of the service suitable starting point in the process of designing service-oriented architecture is service. Once the final list of services was prepared, based on the logic that the service could have been making capsules, the potential use of this logic and this logic relationship with business organizations in various fields, are classified. Classified into three types of public service there are different models that show the service is service oriented architecture projects.

\section{7. evaluate the proposed method:}

Existing methods in identifying service problems and weaknesses with their lack of control that are most similar and overlapping services to identify services to evaluate compliance with organizational objectives identified services and service quality characteristics are not controlled. In the proposed method of extracting primary candidate services, the main focus on the shortcomings in the services making the nominations are due to weaknesses of the region, is. Overall service identification process in the proposed method of three-phase service-oriented analysis, evaluation and optimization, and service organization has been formed.

In the first phase (service-oriented analysis) analysis of both top-down approach and bottom-up analysis has been enjoying. In top-down approach to analysis, the main focus on analysis and user case study areas are business organizations. In this section assume that the extraction cases user organizations has been done before and therefore the necessary activities to identify areas of user cases top-down analysis of the proposed method is out. After the user to describe the cases studied, the model user activity relating to each case and plotted on this model, processing activities, decisions, branching and merging independently or combined as a primary service identified in the list of candidate services are placed. In bottom-up analysis approach, operational and non operational functions hereditary systems and studied the organization and used as services in the list of candidate services are placed.

Therefore important that the first phase of activities is achieved in the proposed method is that of creating a similar service is prevented and the incidence of overlap in service workflow logic is minimized. Perform the necessary control to correct for this service when placing services in the list of extracted candidates, takes place.

In the second phase (evaluation and optimization), the main focus on compliance with organizational goals and nominee services, service quality candidates is based on principles of service orientation. Beginning in the first part of the tree is modeled organizational goals, then provide target matrix - service to identify the needs of each service and the relationship between goals and objectives in the tree is established.

The most important conclusion in this section of the second phase of the proposed method is that is received, we can easily objectives that no service has been assigned to them or vice versa, some service toward the goal of any symptoms have gone, be identified.

In the second part of the second phase, service quality characteristics are studied and evaluated. These features, based on four primary principles of service orientation in the Present Stage Consumer services are identified, are evaluated. The important result of implementing this part of the second phase is that of service total at least recognized principles of service orientation acceptable feature of the four primary capabilities reuse, loose connections, combining flexibility and autonomy. In the third phase (organizing), candidate services as final services were introduced and are proportional to the potential in the three layers of business services, application services and basic services are classified.

To evaluate the optimum method proposed appropriate questions based on qualitative criteria were considered. Considering the questions of quality and effectiveness of design processes, 
activities and methods of forecasting activities in different parts of the proposed method for identifying services provided and the business is set.

More questions intended to evaluate the proposed method is presented:

- For extracting primary candidate services through analysis of user cases and activity diagrams.

- For the nominee services through existing systems of organization functions

- For controlling and preventing the creation of similar and overlapping services between the service

- recall presented two methods for assessment of candidate services including "compliance of candidate services with organizational goals" and "quality of service evaluation"

- Methods and criteria provided in order to analyze and evaluate the goals

- Rules of procedure provided for modeling purposes the tree

- Determine objectives covered by the service using the service matrix and objective evaluation

- For solving potential problems in the allocation of services to organizational goals

- Quality and comprehensiveness criteria presented (the ability to reuse, loose connection, autonomy, and combining services) to assess

- For controlling and evaluating the service quality characteristics

- Quality of proposed measures to address potential problems in service quality characteristics •

Method was introduced to organize and classify services

- Quality model as a general process

In order to evaluate the possibility of providing the above criteria, a questionnaire including summary descriptions of the proposed method and the evaluation form was prepared and delivered to the software experts in the field was placed.

Summarize the main conclusion of the expert opinions and analysis are receiving is that the proposed method with a high percentage of accepted analysis and design software experts in the field located and acceptability is high.

\section{Conclusion}

Initial integration of technologies, mainly problems of heterogeneity or promote flexible applications had to overcome, but today's complex business environment, solutions need to tolerate the failure to ensure their service and also provide the necessary agility. Here, the purpose of agility, by monitoring the burst and the market demands the ability to respond quickly to this demand by offering new products is the orientation of integration of data applications that can manage them, they went into service some the quality, accessibility, fault tolerance and security, is guaranteed by applications. The need for integration, software architecture orientation of the core application oriented organization has changed. Therefore, software architecture components of fine grain objects and components, the services have developed coarse. In conclusion, the use of SOA instead of object-oriented architecture and component-based has proven to be better.

\section{REFERENCES}

[1] Easwaran,G and Nadhan. "Service-Oriented Architecture: Implementation Challenges":Microsoft Architect Journal, 2004

[2] Qinying Li, "Bank Application System Integration Based on SOA and EAI," Intelligent Information Systems, IASTED International Conference on, pp. 271-274, 2009 International Conference on Industrial and Information Systems, 2009: http://doi.ieeecomputersociety.org/10.1109/IIS.2009.111

[3] Roshen.W, SOA-Based Enterprise Integration: A Step-by-Step Guide to Services-Based Application Integration. 2009. The McGraw-Hill Companies.

[4] Gulledge T. (2006) What is integration? Industrial Management \& Data Systems, 106, 5.

[5] Erl, T. (2008). [Chapter 4]. In SOA Principles of Service Design. Prentice Hall.

[6] Mahmud, Q. H. 2005, Service-Oriented Architecture (SOA) and Web Services: The Road to Enterprise Application Integration (EAI),

[7] Lam W. (2005) Barriers to e-government integration. The Journal of Enterprise Information Management, 18, 511. 
Advanced Computing: An International Journal ( ACIJ ), Vol.2, No.5, September 2011

[8] Qureshi K.A. (2005) Enterprises Application Integration. In: IEEE International Conference on Emerging Technologies, Islamniabad. [9]

oldooz karimi holds a BSc on Software Computer Systems and an MSc in the Software Computer sign, both from the University of AZAD. 1. Her research interests include Service, SOA, Enterprise, Enterprise architectures and Security. She has written extensively in these areas. She can be contacted on oldooz_karimi@yahoo.com.

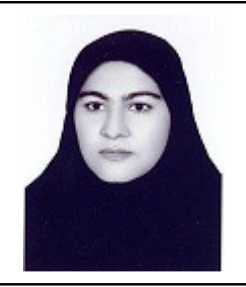

